\title{
When Transportation Meets Communication: V2P over VANETs
}

\author{
Nianbo Liu*, Ming Liu*, Jiannong Cao ${ }^{\dagger}$, Guihai Chen ${ }^{\ddagger}$ and Wei Lou ${ }^{\dagger}$ \\ ${ }^{*}$ School of Computer Science and Engineering \\ University of Electronic Science and Technology of China \\ Email: liunb@uestc.edu.cn, csmliu@uestc.edu.cn(corresponding author) \\ ${ }^{\dagger}$ Internet and Mobile Computing Laboratory \\ Hong Kong Polytechnic University \\ Email: csjcao@comp.polyu.edu.hk,csweilou@comp.polyu.edu.hk \\ ${ }^{\ddagger}$ Department of Computer Science and Engineering \\ Shanghai Jiao Tong University \\ Email: gchen@nju.edu.cn
}

\begin{abstract}
Information interaction is a crucial part of modern transportation activities. In this paper, we propose the idea of Vehicle-to-Passenger communication (V2P), which allows direct, instant, and flexible communication between moving vehicles and roadside passengers. With pocket wireless devices, passengers can easily join VANETs as roadside nodes, and express their travel demands, e.g., taking a free ride or calling a taxi via radio queries over VANETs. Once a matched vehicle is found through the disseminated queries, the driver can decide whether to provide corresponding services, especially the carrying of passengers and goods. We investigate the main challenges in vehicle calling, establish a trip history model to predict vehicle movement, and develop typical query dissemination schemes to match the target vehicle in vehicular networks. With V2P over VANETs, vehicle transportation is capable of open and efficient P2P information interaction, and thus benefits from relevant efficiency improvement. Based on a realistic travel survey and simulation, we prove that vehicle calling is effective and efficient in casual carpooling and taxicalling.
\end{abstract}

Keywords-VANET; V2P; carpool; ride-sharing

\section{INTRODUCTION}

The efficiency of vehicle transportation indicates how we utilize vehicles and also, the degree of expensive social costs, such as traffic jams, carbon emissions, pollution, accidents, energy crisis issues, and other problems. Although lots of institutions, resources, and researches are dedicated to improving transportation efficiency, the waste of transportation abilities is still ubiquitous in current vehicle transportation.

According to NHTS [1] from the U.S. Department of Transportation, the average occupancy rate of personal vehicle trips is 1.6 persons per vehicle mile. Since a regular vehicle carries 5 persons in full occupancy, $68 \%$ of transportation abilities are wasted during personal trips. In the U.S. alone, this involves 204 million personal vehicles and causes a great deal of loss. Similarly, such inefficiency has also been observed in business transportation. Taxies, vans, trucks, and other vehicles are often running in low occupancy or utilization, or are sometimes even unoccupied. The oversupply doesn't just come from wasted transportation abilities, but also comes from information opacity between supply and demand. Unfortunately, current interaction methods between moving vehicles and passengers are awfully poor, both in techniques and results. A small portion of taxies and trucks are equipped with onboard wireless stations, but the communications are usually limited in small vehicle groups. In the traditional "thumb a ride" method, drivers need to stop for passengers gesturing at them just to have a face-toface talk. It is restricted in sight, often fails if the driver and passenger disagree, and has been prohibited in many places due to safety concerns. Many transportation enterprises, call centers, carpool associations, and other institutions try to provide information support and transportation arrangements for vehicles and passengers, but the centralized services are not scalable for the extensive, rapid-changing, and highly diverse transportation activities. Some administrations have introduced High Occupancy Vehicle (HOV) lanes to encourage ride-sharing. Casual carpools, as impromptu carpools formed among strangers, can team up in public areas near HOV lanes [2], but it is severely limited in deployed roads.

Vehicular Ad Hoc Networks (VANETs) are emerging as a new technology, integrating the capabilities of new generation Peer-to-Peer (P2P) wireless networks with vehicles. Vehicles are equipped with wireless devices that allow them to exchange messages with each other as Vehicle-to-Vehicle communication (V2V). They can also exchange messages with roadside infrastructures as Vehicleto-Infrastructure communication (V2I). The application area ranges from safety-related warning systems to improved navigation mechanisms, as well as information and entertainment applications. However, such applications and services are provided for drivers and occupants in moving vehicles, without involving any potential passengers at roadside. It makes the information opacity between supply and demand of transportation remain ignored in vehicular communication. Thus, V2V and V2I cannot offer any additional advantages to the waste of transportation abilities, and the inconvenience of roadside passengers. 
In this paper, we propose the idea of V2P, which allows direct, instant, and flexible communication between vehicles and roadside passengers. With pocket wireless devices, passengers can easily join VANETs as roadside nodes and express their travel demands, e.g., taking a free ride or calling a taxi via radio queries over VANETs. Once a matched vehicle is found through the disseminated queries, the driver can decide whether to provide corresponding services, especially the carrying of passengers and goods. We investigate the main challenges in vehicle calling, establish a trip history model to predict vehicle movement, and develop typical query dissemination schemes to match the target vehicle in vehicular networks. With V2P over VANETs, vehicle transportation is capable of open and efficient P2P information interaction, and thus benefits from relevant efficiency improvement. Based on a realistic travel survey and simulation, we prove that vehicle calling is effective and efficient in casual carpooling and taxi-calling.

The major contributions of this work are as follows. (1) We firstly facilitate a combination of transportation and communication in VANETs. Once passengers join the communication, VANETs can be regarded as a distributed information platform for the cooperation among people with transportation tools and transportation objects. (2) We refer to the non-random nature of vehicle movement, and establish node-specific vehicle mobility models at the microscopic scale. Vehicular communication can be built on a prior knowledge of vehicle movement from the moving history of individual vehicles. (3) We develop practical vehicle calling schemes, especially the revolutionary casual carpooling without service center. As a result, people can easily access any available ride-sharing at any time and any place in traffic networks.

The remainder of this paper is structured as follows: Section II presents a brief overview of related work while Section III introduce V2P and vehicle calling. In Section IV, we analyze the transportation service and the vehicle mobility model. Section $\mathrm{V}$ focuses on query dissemination, and Section VI discusses implementation issues in vehicle calling. Section VII evaluates vehicle calling via a survey and simulation. Finally, Section VIII summarizes the paper, and also outlines the research perspectives.

\section{RELATED WORK}

There have been many studies on information system and transportation interaction in vehicular communications. In this section we only explore the most relevant work in the areas.

In recent years, Intelligent Transport Systems became a hotpoint for the VANET community. Many schemes were proposed to deal with traffic problems through vehicular communication, such as CarTel [3] and MobEyes [4]. These approaches mainly concentrate on congestion control through V2V and V2I interacting, but haven't taken transportation in itself into consideration. However, some research introduces new peers into VANETs. FleaNet [5] allows some roadside nodes with wireless devices, such as pedestrians and shop owners joining vehicular communication. It makes VANETs become the concept of a virtual "flea market" as a distributed platform for the cooperation among people with shared interests. Despite no transportation consideration, the idea of FleatNet contributes some illuminations to our work: pedestrians can join VANETs as roadside nodes; mobile and stationary users, no matter if they are drivers or pedestrians, can express their demands and search target resources over VANETs.

In order to improve transportation efficiency, some researches [6], [7], [8], [9] try to develop ride-sharing systems without HOV limit, which provide dynamic travel matching by extending current cell phone services or web-based ones. Similar to current taxi call center, background systems trace the movement of vehicles, and then inform passengers and drivers of nearby objectives according to their queries. However, such systems has their own problems. First, the service charge is an obstacle to the users. Second, the use of cell phones while driving is illegal in many countries, and is also a major cause of accidents. Finally, the centralized framework is not scalable for extensive transportation activities. Tracing all moving vehicles in cities or highways can be highly expensive, while reducing the tracing vehicle numbers will degrade the quality of the service. Thus, our work discards service center and vehicle tracing, establishes distributed V2P communication and local matching as a cheap and scalable solution.

\section{V2P AND VEHICLE CALling}

For the idea of V2P, we first explain the background and connotation, and introduce vehicle calling as a practical implementation. Then, we propose a typical vehicle calling process, and analyze major challenges in the process.

\section{A. V2P in Traffic Networks}

Mail delivery by horse-drawn carriages and other vehicles has been used for centuries, in which communication depends on actual mail transportation, traditionally. The invention of the telegraph, telephone, and Internet led to new information carriers, and then successfully separated communication from transportation. Up until recently, the VANET community began to pay much attention to moving vehicles, as the focus was mainly on communication networks based on mobile vehicle nodes. At this time, transportation meets communication again when it comes to vehicles, because vehicles not only transport passengers and goods, but also carry and forward data packets through wireless devices. Fig. 1 describes the layered functionality abstraction of realistic traffic networks, in which a combination of transportation and communication is taken into account. 


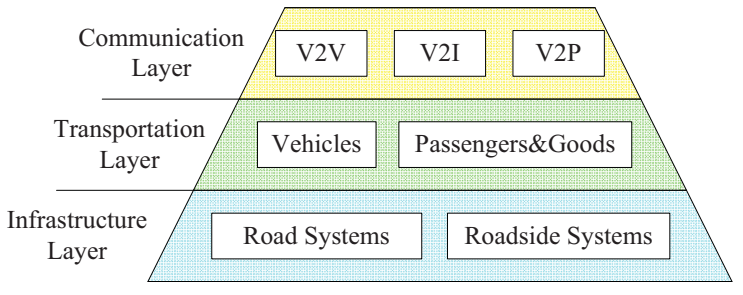

Figure 1. Layered traffic networks

Infrastructure Layer: Road systems contain many infrastructures, such as roads, intersections, traffic signs, traffic lights, power supplies, bus stops, and so on. Roadside systems mainly involve roadside units for communication, such as sensors, access points, central units, wired and wireless connections, etc.

Transportation Layer: Since no central entity could or should organize transportation activities, traffic participants, including vehicles (drivers), passengers, and goods, are self-organized in a distributed manner for the support of passenger and freight transportation.

Communication Layer: V2V and V2I focus on safety and comfort applications for moving vehicles, whereas V2P supports transportation applications for vehicles and roadside passengers.

As shown in Fig. 1, the traffic flow, information flow, and passenger and commodity flow are interrelated and interact with each other in traffic networks. VANETs permit mobility-assisted routing so that vehicles can carry packets, and forward them across network partitions, e.g., mail transportation. These carry-and-forward schemes reflect the fact that vehicular communication benefits from actual transportation. Thus, we suggest a reserve approach to promote passenger and freight transportation by vehicular communication in a P2P manner, as V2P over VANETs.

\section{B. Vehicle Calling}

Transportation involves many activities and participants, which may require highly complex information interaction among vehicles and passengers. In this study we only discuss the simplest demand-driven V2P interacting as vehicle calling, which focuses on how to get a vehicle for roadside passenger through vehicular communication. Here we treat goods as a special kind of "passenger", for goods owners can attend V2P and call vehicles for freight transportation.

In V2P, both vehicles and passengers need necessary hardware equipment to support the communication. When it comes to vehicles, GPS and electric maps are widely deployed, as well as wireless devices. Since there's no standard human-machine interface, we assume that some signal lights, buzzers, or graphical or phonetic interfaces can inform the driver of vehicle calling, allowing the driver to answer yes or no by pushing buttons on the device. To individuals, previous wireless mobile devices, such as cell phones, i-Pods, PDAs, and laptops, are not designed for vehicular communication. So, we assume that passengers have pocket wireless devices as vehicle callers in order to perform this task, which can be regarded as a cheap device with an electric map and simple input/output.

As shown in Fig. 2, a typical vehicle calling has the following steps:

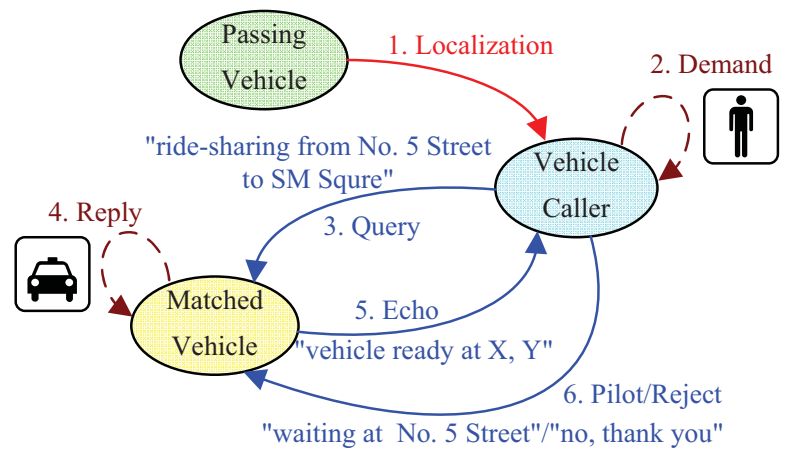

Figure 2. A typical vehicle calling

1. Localization: once activated, a vehicle caller starts to monitor nearby beacon signals in order to locate its rough position from the GPS locations of passing vehicles.

2. Demand: a passenger customizes a vehicle calling according to his/her requirements, and then distributes the message over VANETs.

3. Query: vehicles are checked on calling requirements during the dissemination of the query message, with which some matched vehicles may be found.

4. Reply: a driver is informed of the calling via an onboard device, which he can then decide to accept or refuse/ignore the calling.

5. Echo: if the driver accepts the calling, an echo message is sent to the passenger.

6. Pilot/Reject: if the echo message is the first one, or is validated by the passenger, the vehicle caller keeps sending pilot messages until the vehicle stops for the passenger. If not, it sends a reject message to the vehicle.

There are several challenges in the implementation of this idea. First, vehicles need to provide explicit services and transparent matching without driver interaction, in which the key content lies in the describing and modeling of vehicle characteristics. Second, query dissemination over VANETs should be adaptive to complex environments, efficient in delivery, and lightweight in overhead. Finally, social and economic problems in vehicle calling should be considered, as well as technical ones.

\section{Vehicle Description}

In this section, we focus on the description of vehicle in V2P. So the transportation services of vehicles are investigated, and the node-specific mobility model is discussed in theory and practice. 


\section{A. Transportation Service}

From the viewpoint of traffic networks, VANETs can be regarded as heterogeneous transportation networks, in which vehicles could explicitly advertise their respective types and transportation services for vehicle calling.

Table I qualitatively lists some vehicles tightly related to our life. Buses and subways support public transportation on some predefined routines. Taxies and trucks provide business services for passengers and goods. Ambulances, police cars, fire trucks, and other special vehicles respond to emergency calling, while personal vehicles take up carpools in their respective trips. Generally, we can divide their transportation services into two categories: clean state and ride-sharing. In the clean state service, vehicles are unoccupied and ready to run for any passengers. In the ride-sharing service, vehicles have predefined trip (no matter for driver, first passenger or public) so that they only pick up the passengers on their way. Emergency-used vehicles must respond to clean state calling as quickly as possible. Buses and personal vehicles have predefined trips so that they only provide ride-sharing along their routes. The services of taxis and trucks are often dual: if unoccupied, they are ready to serve new customers without any predefined trips; if the fulfilled task is not exclusive, they are glad to pick up more passengers or goods on their trips, for profit.

Table I

VEHICLE AND TRANSPORTATION SERVICE

\begin{tabular}{|c|c|c|c|}
\hline Vehicle Type & Purpose & $\begin{array}{c}\text { Clean } \\
\text { State }\end{array}$ & $\begin{array}{c}\text { Ride- } \\
\text { sharing }\end{array}$ \\
\hline \hline bus, subway & public & no & yes \\
\hline taxi, trunk & business & yes & yes \\
\hline ambulance, etc. & emergency & yes & no \\
\hline personal vehicle & personal & no & yes \\
\hline
\end{tabular}

For the support of vehicle calling, a vehicle should add the following contents in its beacon: vehicle type, moving destination, ready for clean state service, ready for ridesharing service. Vehicle type is fixed to a certain vehicle. The moving destination indicates the target position of the current moving vehicle. An unoccupied vehicle may have no certain moving destination. In order to avoid undesired calling, drivers can declare being equipped with or out of clean state and ride-sharing services via an onboard device that allows or refuses query messages from VANETs. In the clean state service, passengers should appoint vehicle type, service type, and current position in the query. In the ridesharing service, the passenger's travel destination should be attached to the query so that the vehicle can check whether the passenger's position and destination are located in its prospective route. In Fig. 2, a carpool at No. 5 Street may send a query as, "ride-sharing from No. 5 Street to SM Square". It means that a ride-sharing vehicle, which is going to pass by No. 5 Street and SM Square, should be matched in query dissemination.
Different vehicles can acquire knowledge of self movement in different ways. Since buses have scheduled routines, the moving destination is available from predefined trajectories. To taxis and trucks, drivers have a profit incentive to input the travel destination into the onboard device in order to afford more ride-sharing services. However, personal vehicle drivers do not have the habit of inputting travel destinations into onboard devices during their trips, unless they need navigation. How to predict the moving destination of personal vehicles becomes a key problem in casual carpooling.

\section{B. Vehicle Mobility}

The researches on human mobility were developing rapidly in recent years. Some of them notice the relationship between human social activities and geographic movements. The time-variant community mobility model [10] captures two properties of human mobility via empirical WLAN traces: skewed location visiting preferences and periodical re-appearance of nodes at the same location. Another study [11] investigates the trajectories of 100,000 anonymous mobile phone users, and finds that human trajectories show a high degree of temporal and spatial regularity: each individual can be characterized by a time independent characteristic length scale, and a significant probability to return to a few highly frequented locations.

According to NHTS, the majority of individual daily trips - 87 percent - are taken by personal vehicle. The daily activities of an individual person, including "going to work", "having lunch", "shopping", and so on, often show regular features. As a kind of human activity, driving is controlled by individual drivers, and follows their respective social activities, partially. Although some unexpected driving occurs, individual or household driving in a certain vehicle usually yields the same spatial and temporal features. For instance, a commuter always drives his/her car from home to office at 9:00, and from office to home at 17:00. It makes large amounts of vehicle movement predictable at the microscopic scale. Research in the field of transportation also validates the regularities, both in human mobility and vehicle mobility. The Mobidrive work [12], [13] monitors the trajectories of private cars by collecting their GPS data. A mobility pattern is observed in Fig. 3, which is constituted by the spatial distribution of those locations where a traveler has had six weeks of personal experience. The spatial regularities in vehicle mobility are marked by the grey lines of vehicle movement, in which two to four main locations (including home) cover more than $70 \%$ of the overall trips.

Some mobility prediction schemes, PGR [14] and the car navigation system [15], try to establish the mobility pattern in Fig. 3 in an individual node by recording node-specific trajectories from GPS data. With trajectory history, a moving vehicle can compare its position to its previous trajectories, finding out the most possible route. However, such 


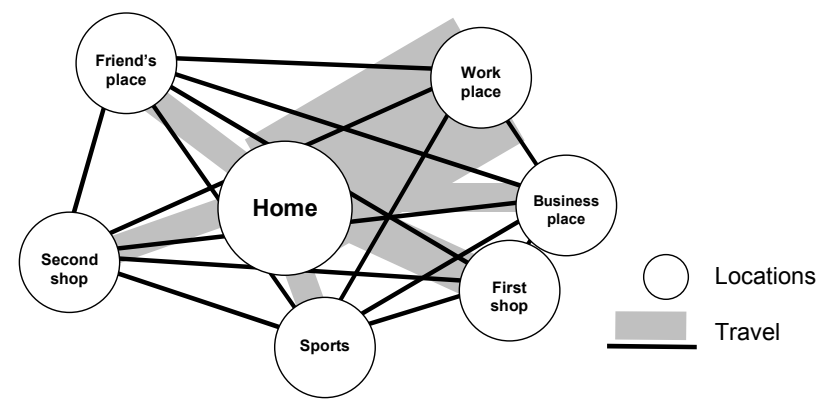

Figure 3. Mobility pattern in Mobidrive

trajectory-based patterns also have some intrinsic drawbacks. First, the time criterion in vehicle movement is completely neglected. If a driver starts his/her car at home in a workday morning, he/she is driving to the work place most likely. If he/she does so in a non-workday evening, the possibility he/she is going to the work place is very slim. Since the trip history is simplified to the trajectory history, the temporal characteristics of vehicle movement are inevitably lost. Second, the overlapped trajectories can cause prediction errors. If a vehicle is moving at the only path to the driver's home, the route is clear. If the path leads to his/her home and favorite shop, the destination prediction by simple route pruning is questionable. Finally, the trajectory records bring a heavy burden in data processing and storage, especially those from long-distances or infrequent trips. Discarding these records may result in false predictions, whereas keeping them will exceed the capacity of the onboard device.

Thus, we need a new mobility model to predict vehicle movement, which should be spatio-temporal in mobility features, doubtless in the destination prediction, and lightweight in data size.

\section{Trip History Model}

Here we explain the idea of trip history by giving a simple example. In Fig. 3, a series of trips taken by the driver can be kept as records in Table II, in which temporal and spatial features of the trips are more concisely represented.

1. Drive from home to work place at 8:40, Friday.

2. Drive from work place to home at 17:35, Friday.

3. Drive from home to sports at 14:05, Saturday, and send his wife to first shop on the way.

4. Drive from sports to friend's home at 18:55, Saturday.

Table II

TRIP HISTORY RECORD

\begin{tabular}{|c|c|c|c|c|c|}
\hline & Day & Time & Source & MP & Destination \\
\hline \hline 1 & Fri. & $<10$ & home & & work place \\
\hline 2 & Fri. & $16-18$ & work place & & home \\
\hline 3 & Sat. & $14-16$ & home & $*$ & sports \\
\hline 4 & Sat. & $>18$ & sports & & friend's home \\
\hline \multicolumn{7}{|c|}{ *first shop is the Midway Point of the third trip. }
\end{tabular}

The locations "home", "work place", "sports", and "friend's place" are not accurate GPS positions, but rough regions in geography. Since the driver may have no special parking space in these frequent visiting places, nearby parking positions within a certain scope, e.g., within $500 \mathrm{~m}$ can be regarded as the same parking location of a given place. Similarly, the start time of the trip is separated into discrete time sets: Day (Mon., Tues., Wed., Thurs., Fri., Sat., Sun.) and Time $(<10,10-12,12-14,14-16,16-18,>18)$. Whenever a driver starts his/her car, the onboard device will log the start time and position as "Day", "Time", and "Source". During movement, it obtains continuous position data from the GPS every several seconds. When the car stops, the last position becomes "Destination". After finishing a trip, the device can abbreviate the trip trajectory. If the trajectory from Source to Destination accords with the shortest path (not zero) in the electric map, it cancels all middle position records as record 1, 2, and 4. If the trajectory doesn't accord, or Source equals Destination, it tries to find a shortest path from Source to later positions for as long as possible, cancels the middle positions from Source to the first Midway Point, and repeats the procedure until arriving at Destination. Record 3 indicates such abbreviation, in which the Midway Point can be the first shop. Since most daily driving trajectories take the shortest paths in geography, the trip records can be largely shortened in data size.

With the trip history, we develop a heuristic and contextdependent induction method based on decision trees, to predict vehicle moving trajectories. In data mining and machine learning, decision trees are widely used as the predictive tool mapping from observations about an item, to conclusions about its target value. The related theories and algorithms can be found in [16]. When a car starts, it constructs a decision tree, where previous trip records in Table II are expressed as branches and leaves in Fig. 4. In each leaf node, the probability of selecting a destination is given by:

$$
p_{k q}=\frac{f_{k q}}{N_{k}}
$$

where $N_{k}$ is the total number of trips starting at root node $k$, and $f_{k q}$ is the number of trips according with specific time, midway point and destination choices at leaf node $k q$. Since category $k q$ gives a final destination choice, $p_{k q}$ represents the probability of moving toward the destination in history. Since the car knows current Source, Day, and Time values, it can find a Destination choice with a maximum $p_{k q}$ value in the tree as an initiate prediction.

While driving, the car periodically checks whether its position is on the way to the predicted destination or midway point. If the position disagrees with the predicted route, the car needs to calculate a new destination probability by: 


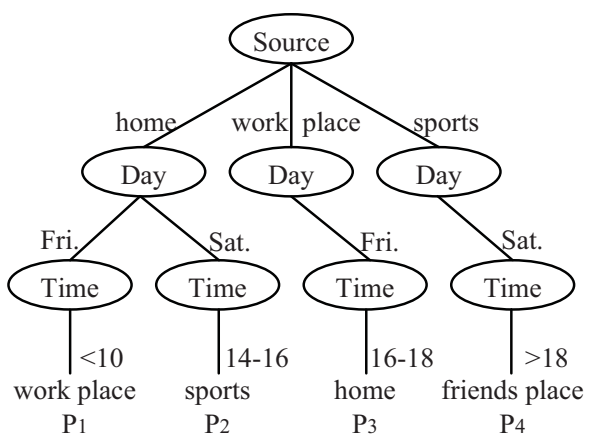

Figure 4. Decision tree structure

$$
p_{k q}=\left\{\begin{array}{cc}
0 & \text { if the route is impossible } \\
\frac{f_{k q}}{\sum f_{k}} & \text { otherwise }
\end{array}\right.
$$

where $\sum f_{k}$ is the total number of the rest of the trips after removing the infeasible ones. Then, it can find a new destination prediction with maximum probability $p_{k q}$. This process can be triggered by the interactions of drivers, likewise. When the onboard device shows up-to-date predictions, the driver can confirm the destination, or deny it to restart a new prediction. Consequently, a vehicle can predict its movement via the trip history model for transparent travel matching.

\section{QUERY DisSEMINATION}

For vehicles ready to provide services, passengers launch vehicle calling to search and access proper transportation. Most emergency-use vehicles are prone to swarming at specific locations, such as ambulances at hospitals and police cars at police stations. Public vehicles are running on scheduled routines. Although passengers could call these vehicles through point-to-point and source routing queries, current call centers or bus tracking systems provide similar services with real-time response. So we mainly focus on the calling for "ordinary vehicles", those taking up the major part of traffic and lacking access service.

In regards to business and personal vehicles, there is no determined destination for vehicle calling. But, some calling may occur between passengers and vehicles within mutual radio range. Since the vehicle caller can monitor nearby beacons, query messages can be directly sent to a matched vehicle. If the radio range is $250 \mathrm{~m}$, the driver has enough time to respond to the calling because most vehicles have a braking distance (from 100kph to 0) within $100 \mathrm{~m}$. Then, the driver can stop for the passenger via the short-haul navigation of pilot messages. Consequently, passengers can easily call any passing vehicles and access their services without extra query dissemination. This enables simple and facile ride-sharing for carpools at roadside.
In regards to the vehicles outside the radio range, we need a proper strategy to spread queries over VANETs to match target vehicles. Epidemic routing [17] ensures eventual message delivery via random pair-wise exchanges of messages between mobile hosts, which has decent performance, but often causes heavy resource consumption in the network. FleaNet [5], as a 2-hop delivery approach, only diffuses a query by vehicle carrying, which is very scalable, but not suitable for real-time interacting. Geocast [18] can restrict the transmission of a message to a predefined geographical region, which also incur overmatching in query dissemination. Since the vehicle's GPS knows the exact position, GPSR [19] provides a location-based scheme that all packets transmitted into the network are marked by the originator with their destination's location. In greedy forwarding, intermediate nodes simply choose to forward the packet to the neighbor located closest to the destination, which is efficient and robust in straight roads, but often causes extra transmission and delay in complex topologies. In order to match vehicle quickly and efficiently, we develop an enhanced GPSR to disseminate a query in a neighboring area around a passenger.

In the highway shown in Fig. 6, the passenger sends out queries as GPSR messages from Source to $D_{1}$ and $D_{2}$, the estimated delivery destinations in the electric map. In greedy forwarding, the intermediate node will check all neighboring nodes: if one node matches the query, it sends the message to the target node; if no vehicle matches, it sends the message to the next intermediate node until the message arriving at its destination is discarded. If no echo message returns, the passenger can expand the calling scope and send queries to further destinations.

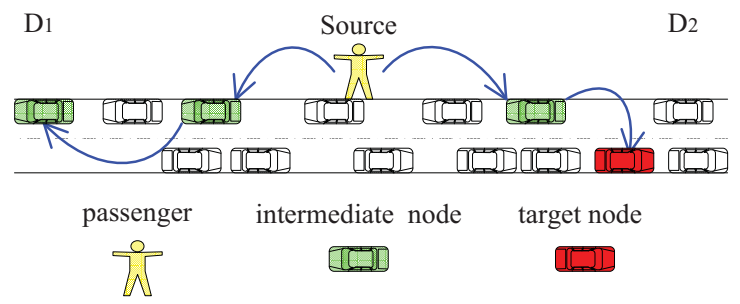

Figure 5. Query dissemination in highway

In the city scenario shown in Fig. 6, query messages should be flooded into nearby streets around passengers within the calling scope. To achieve this aim, the messages from Source are attached to a scope limit and destined for neighbor intersections as $I_{5}$ and $I_{8}$. We assume all intersection positions from $I_{1}$ to $I_{11}$ are marked in the electric map. In later dissemination, the messages arriving at an intersection will be replicated and destined for all neighbor intersections, except the traversed intersection. As shown in Fig. 6, the passenger gradually expands the calling scope when no target vehicle is found: the first calling is in 
the local street within $R_{1}$; the second calling may arrive in the areas near $I_{5}$ and $I_{8}$ within $R_{2}$; the third one may reach all streets in the figure within R3. In order to avoid looping, we add a parameter as the intersection hop to query messages, which indicates how many intersections a message can pass. Whenever a message arrives at the intersection, the value of the intersection hop will subtract one like the TTL in IP packet delivery. If the value equals zero, the message is discarded.

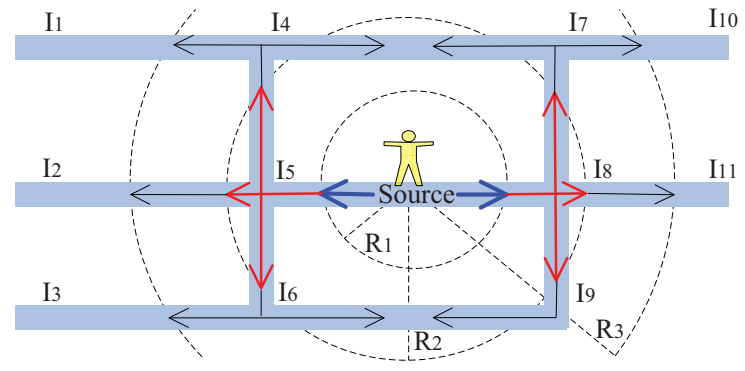

Figure 6. Query dissemination in city

In sparse networks, message delivery in vehicle calling may incur uncertain delay or packet loss. Even if the vehicle is found in the query dissemination, later, echo and pilot/refusal message deliveries may have a long-time delay. It allows a given query to find many redundant vehicles without time consuming validation from the passenger. In this study we assume that the drivers of matched vehicles can respectively decide whether to move toward the passenger according to the positions of two sides, and the delay of query messages. The mechanism to reduce overmatching in query dissemination will be our next research agenda.

\section{IMPLEMENTATION DISCUSSION}

Even though vehicle calling is feasible in technique, the implementation still confronts lots of social and economic problems. As these problems have not been carefully considered, we only provide an initial discussion, and list them as future work.

An obvious obstacle in vehicle calling is the dependence on the vehicle caller. If people are not familiar with V2P, they will not pay for such a device, and the generalization at the initial stage will be difficult. A viable alternative is to install some public vehicle callers in the dense traffic areas as roadside infrastructures so that people can gradually accept vehicle calling. At the same time, incentive mechanisms to encourage vehicle calling are necessary, especially for drivers.

Security and comfort are important factors that deeply concern both drivers and passengers. Strangers in the same vehicle need to have a basic level of trust in order to not be too worried or concerned with possible unpleasant behavior or risks of accidents or violence. For example, some research
[20] deploys individual RFID device to identify the driver and the passenger in casual carpooling. Similarly, proper authentication for individual vehicle caller is needed. According to previous $\mathrm{P} 2 \mathrm{P}$ cooperation experiences, a reputation system is helpful in regulating individual actions in a trustworthy manner. Once the driver and passenger make a deal in the three-way "query-echo-pilot", two sides can respectively send the deal note to the reputation system via VANETs. After finishing a carry, they can send feedback to one another, similarly. If possible, personal information, such as criminal records and driving records, can be added into the system. Deal notes, feedback, and personal information can influence the reputation of vehicles and passengers, and help them avoid unfavorable matches in vehicle calling.

Privacy is another matter that causes a lot of worry. Some drivers and passengers may have a problem with exposing their travel destinations. In this case, accurate travel destinations can be replace by some common transit points, such as intersection or street name. For further anonymity, some encryption and decryption mechanisms should be considered. How to balance privacy and efficiency will be an important subject in vehicle calling.

The actual matching of content in vehicle calling can be highly complex due to distinct travel demands. A carpool may accept a halfway carrying to his/her destination, while a business man may want to bargain with truck drivers. Therefore vehicle calling should be context-based, selfadaptive, and scalable for different transportation activities.

Although involving many problems, vehicle calling also brings some additional advantages. For example, vehicle callers support navigation and trip planning for pedestrians, and trip history models enable "Smart Cars" with auto navigation, explicit message relaying, and other mobilityassisted applications.

\section{TRavel SuRvey and Simulation}

In order to evaluate vehicle calling accurately, we first construct a realistic travel survey to demonstrate the trip history model, and then examine the performance of vehicle calling in casual carpooling and taxi-calling.

\section{A. Travel Survey}

To evaluate the driving regularities for individual vehicles, and the accuracy of our trip history model, a travel survey, taken over 9 weeks, has been performed. Five volunteers from the academic staff were invited to attend the survey, not involving any authors or contributors of this study. Each one processes a private car with GPS. During the survey, they and their family members driving the cars were asked to note down the start time, source position, and destination position of each car, as well as the midway point positions, if there were any. Then, we collected the records and calculated trips for each car in Table 3. We found partial travel numbers from 135 to 243 , due to the different household driving habits. 
And the average trips in each car respectively range from 2.14 per day to 3.86 per day.

Table III

COLLECTED TRIPS IN TRAVEL SURVEY

\begin{tabular}{|c|c|c|c|c|}
\hline Car & $1-8$ week & 9 week & Total & Average \\
\hline \hline A & 155 & 17 & 172 & 2.73 \\
\hline B & 214 & 29 & 243 & 3.86 \\
\hline C & 169 & 18 & 187 & 2.97 \\
\hline D & 180 & 25 & 205 & 3.25 \\
\hline E & 120 & 15 & 135 & 2.14 \\
\hline
\end{tabular}

After the survey, the trip records were translated into discrete results in Table 2, and input into the electric map of Chengdu City, China. All trips outside of the city scope were discarded. In order to evaluate the accuracy of predictions, we established a trip history model with the trips in the first eight weeks as a training set, and validated the prediction with the trips in the last week as a target set. We examined the generated up-to-date predictions at different stages in the vehicle moving process. The hit rate, shown in Fig. 7, demonstrates that the trip history model accords with the actual vehicle moving from $65.83 \%$ at the beginning to $97.04 \%$ at the end of the journey. It proves that the trip history model can provide an accurate prediction of vehicle movement, even when the vehicle first starts moving.

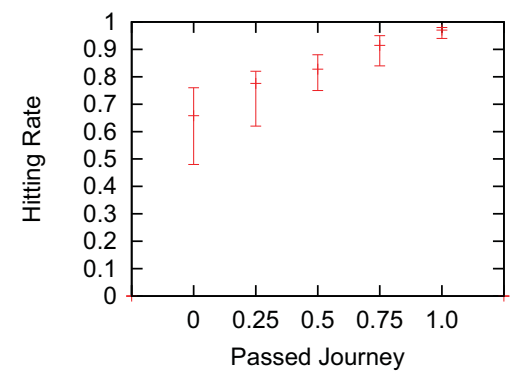

Figure 7. Prediction accuracy

\section{B. Simulation}

As shown in Fig. 8, we extract a regional urban area with the range of $3200 \mathrm{~m} \times 2200 \mathrm{~m}$ from a real street map of Chengdu, which contains 30 intersections and 48 bidirectional roads. Since accurately modeling vehicles' movement is very important for message delivery, we use the open source software, VanetMobiSim-1.1 [21], to generate the movements of vehicles that can be directly utilized by NS2.33. To evaluate the vehicle calling in different traffic density environments, we deploy different vehicle numbers, i.e. $100,200,300$, and 400 , to the map, with the average speed ranges of 40 to 80 kilometers per hour. The radio range is set at $250 \mathrm{~m}$, and the MAC protocol is $2 \mathrm{Mbps} 802.11$. The beacon interval is $1 \mathrm{~s}$, and all messages have a uniform size of $1 \mathrm{~kb}$. In the simulation, we assume that each vehicle has enough of a buffer to prevent message elimination from occurring.

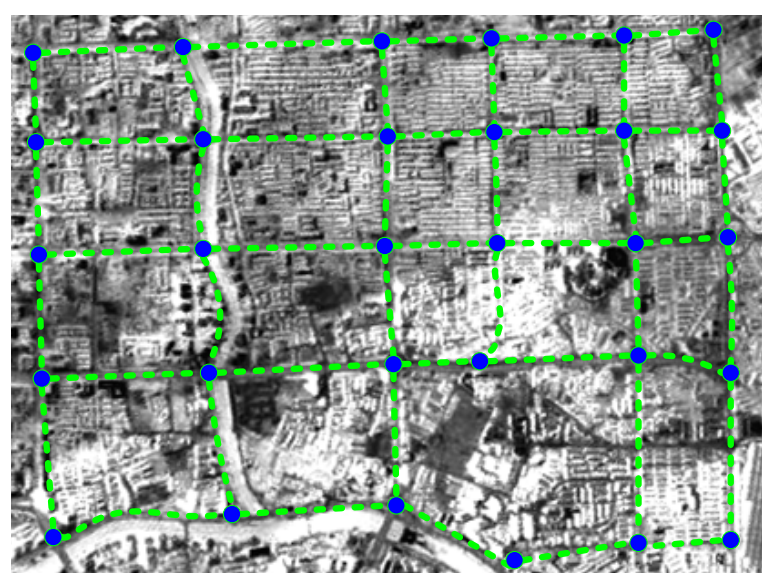

Figure 8. Road topology in simulation

In casual carpooling, a roadside carpool may claim ridesharing of passing vehicles by "thumb a ride" or vehicle calling. We assume that the carpool requests a randomly chosen destination on the road. $60 \%$ of the total vehicles are personal vehicles ready to support ride-sharing, each having a randomly predefined movement destination, but advertising their movement destinations with a correct rate between 0.66 and 0.97 . In vehicle calling, the vehicle caller can actively check nearby beacons, match travel destinations for a carpool, and send queries to the matched vehicles within $250 \mathrm{~m}$. To match an appropriate vehicle, the carpool should pick it up from the average 16-24 passing vehicles as Fig. 9 (a) demonstrates. In "thumb a ride", this means that approximately twenty times, face-to-face talking before a successful matching will occur, which can become so fussy that no drivers or passengers will be willing to do it. It explains the reason why causal carpooling in highways or HOV lanes can never be implemented in urban areas with complex topologies and travel matching. Vehicle calling eliminates face-to-face interacting limits, and achieves favorable calling efficiency: passenger waiting time in different traffic density environments only lasts dozens of seconds in Fig. 9 (b), and average calling is no more than twice in Fig. 9 (c). It makes casual carpooling firstly effective and efficient in any place where vehicles pass by.

In taxi-calling, a passenger can call a taxi in sight by waving his/her hand for it, or they can launch wireless vehicle calling through V2P. We assume that the taxi driver can catch the gesture of a passenger within $100 \mathrm{~m}$, and the scope of vehicle calling can be expanded to $1000 \mathrm{~m}$. In vehicle calling, query messages are disseminated in enhanced GPSR and later echo/pilot messages between vehicle and passenger can be sent in GPSR. With the $10 \%$ ratio of unoccupied taxies to total vehicles, we examine the two methods in simulation and find the passenger waiting time in Fig. 10 


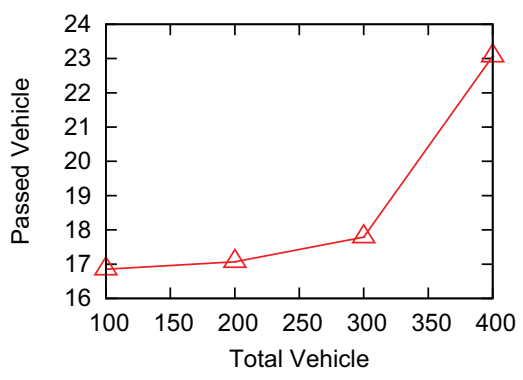

(a) passed vehicle

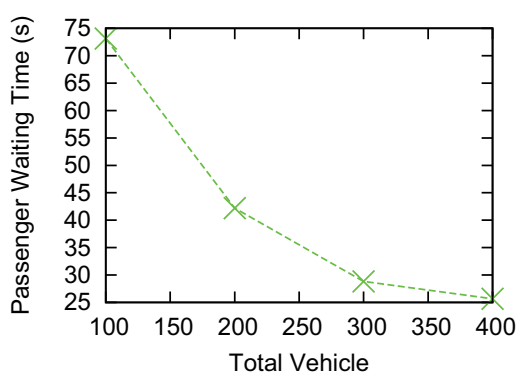

(b) passenger waiting time

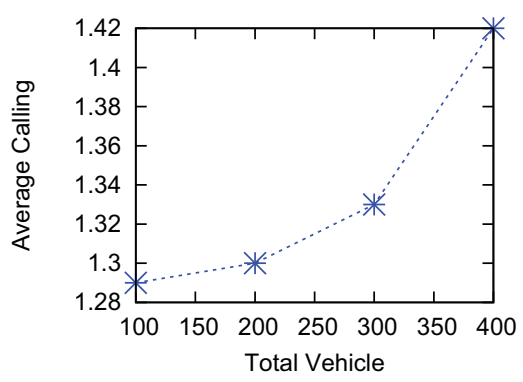

(c) average calling

Figure 9. Vehicle calling in casual carpooling

(a). If the passenger waves his/her hand, a taxi driver may find him after 61-220 seconds. If the passenger uses a vehicle caller, at least one taxi will be informed within 20 seconds. The deliveries of later echo and pilot messages are shown in Fig. 10 (b) and (c). In low traffic density environments the data delivery ratio is very limited, therefore, taxi drivers have to make decisions without having a timely reply. For successful deliveries, transmission delay within 5 seconds is still acceptable for the driver and the passenger.

As shown in Fig. 6, a passenger can gradually expand the calling scope to match nearby target vehicle first. We exam the message delivery with different calling scopes, including $500 \mathrm{~m}, 1000 \mathrm{~m}$ and $1500 \mathrm{~m}$, in vehicle calling. In Fig. 11 (a), the chance of matching is rapidly increase with the calling scope. To the delivery of echo and pilot message In Fig. 11 (b) and (c), expanding calling scope is positive, but the overall delivery ratio is still limited. A passenger can find target vehicle in a large area more easily, because more vehicles are provided in query dissemination. But less traffic can counteract the influence, passengers in low traffic areas have to launch more calling to catch possible vehicles.

Generally, experimental results show that vehicle calling provides simple and efficient vehicle-to-passenger interactions, no matter it's a ride-sharing service or clean state service. Although the calling response is not real-time, the average waiting time of short-range queries is tolerable for users.

\section{CONCLUSION}

We propose V2P to achieve instant and flexible vehicleto-passenger interactions for transportation. The basic idea of $\mathrm{V} 2 \mathrm{P}$ and vehicle calling is simple: if passengers can call any taxi in the street by waving a hand, why not let them call any wanted vehicle in VANETs via a wireless device?

In this paper we introduce passengers to a vehicle caller as static nodes, and then develop VANETs as distributed information platforms for transportation cooperations. In VANETs, moving vehicles, infrastructure, and roadside passengers communicate with each other to support passenger and freight transportation, collectively. Founded with realistic travel demands in mind, V2P contributes revolutionary vehicle calling, enhances vehicle transportation from down to top, and promotes vehicular research, both in depth and scope.

We believe V2P has a bright future in next-generation traffic networks. In such networks, technology is no longer a bottleneck for vehicle-to-passenger interactions, ride-sharing is widely deployed through distributed vehicle calling, passengers can access vehicles on roads as easily as people access electric power from power networks, vehicles are running in high occupancy and less traffic, and all of us benefit from green and efficient vehicle transportation.

\section{ACKNOWLEDGMENT}

The authors would like to thank Haigang Gong, Yong Feng, and the anonymous reviewers for their helpful comments on an earlier draft of this manuscript. This research is supported in part by the China NSF grant (60703114, 60825205, 60903158), the China 863 Project (2006AA01Z198, 2007AA01Z443), the China 973 project (2006CB303000), and the Hong Kong RGC GRF grant (PolyU5102/08E, PolyU5243/08E).

\section{REFERENCES}

[1] "National household travel survey," 2001. [Online]. Available: http://www.bts.gov/programs/national_household_ travel_survey

[2] M. Burris, J. Winn, and W. Associates, "Slugging in HoustonCasual Carpool Passenger Characteristics," Journal of Public Transportation, vol. 9, no. 5, p. 23, 2006.

[3] B. Hull, V. Bychkovsky, Y. Zhang, K. Chen, M. Goraczko, A. Miu, E. Shih, H. Balakrishnan, and S. Madden, "CarTel: a distributed mobile sensor computing system," in Proc. of ACM SenSys 2006. ACM New York, NY, USA, 2006, pp. 125-138.

[4] U. Lee, B. Zhou, M. Gerla, E. Magistretti, P. Bellavista, and A. Corradi, "Mobeyes: smart mobs for urban monitoring with a vehicular sensor network," IEEE Wireless Communications, vol. 13 , no. 5, p. 52, 2006. 


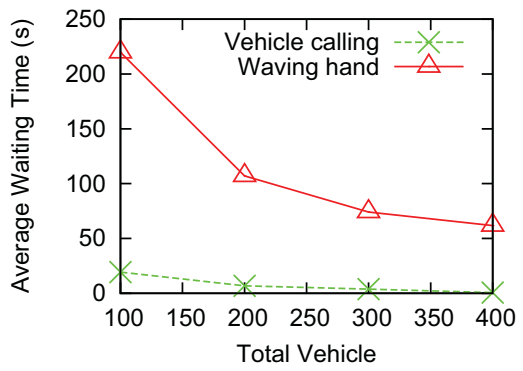

(a) waiting time

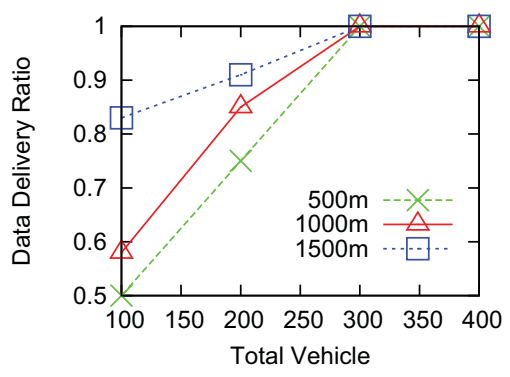

(a) query message

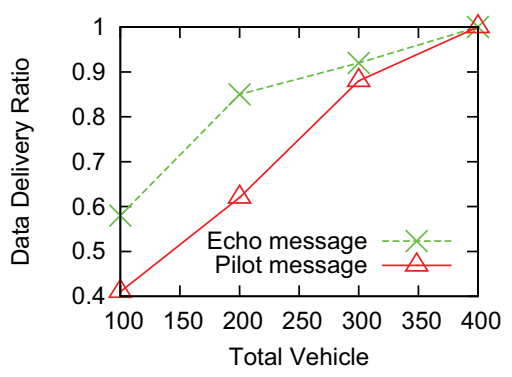

(b) data delivery ratio

Figure 10. Vehicle calling in taxi-calling

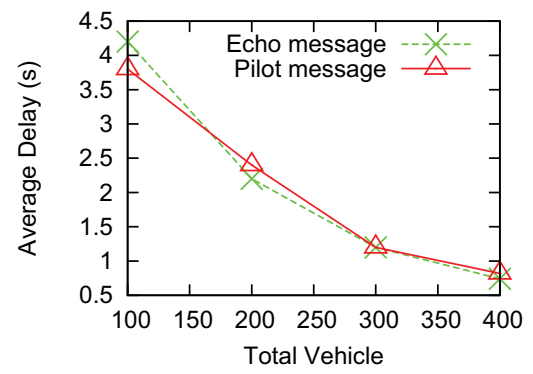

(c) transmission delay (b) echo message

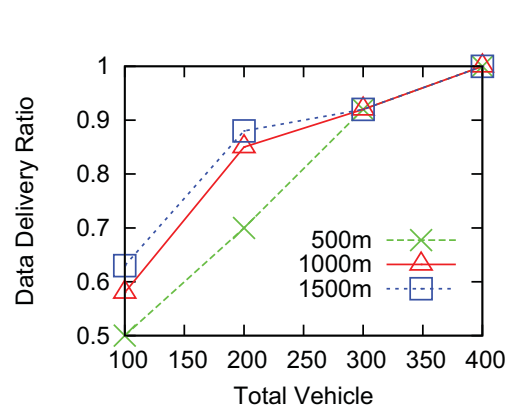

Figure 11. Message delivery with different scope varying

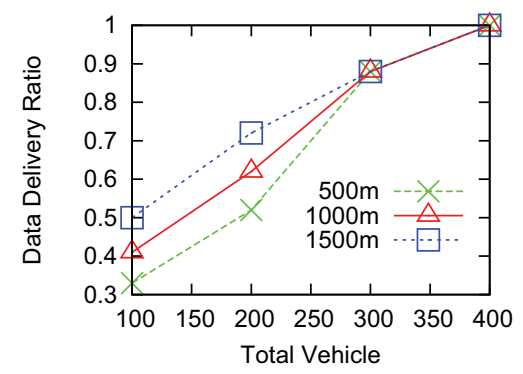

(c) pilot message
[5] U. Lee, J. Park, E. Amir, and M. Gerla, "FleaNet: a virtual market place on vehicular networks," in Proc. of Mobiquitous'06, 2006.

[6] S. Hartwig, "Empty Seats Travelling," Nokia Research Center, Tech. Rep., 2007.

[7] Y. Fu, Y. Fang, C. Jiang, and J. Cheng, "Dynamic Ride Sharing Community Service on Traffic Information Grid," in Intelligent Computation Technology and Automation (ICICTA), 2008 International Conference on, vol. 2, 2008.

[8] A. Lue and A. Colorni, "A software tool for commute carpooling: a case study on university students in Milan," International Journal of Services Sciences, vol. 2, no. 3, pp. 222-241, 2009.

[9] S. Plan, S. Committees, P. Committees, L. Performance, M. Board, T. Coverage, and T. Serials, "Wireless Networks for Car-and Ridesharing Systems: Assessment of $802.11 \mathrm{Wi}-$ Fi," Transportation Research, vol. 9, p. 56AM, 2009.

[10] W. Hsu, T. Spyropoulos, K. Psounis, and A. Helmy, "Modeling time-variant user mobility in wireless mobile networks," in Proc. of IEEE INFOCOM 2007, 2007, pp. 758-766.

[11] M. González, C. Hidalgo, and A. Barabási, "Understanding individual human mobility patterns," Nature, vol. 453, no. 7196, pp. 779-782, 2008.

[12] S. Schönfelder and S. Raumplanung, "Some notes on space, location and travel behaviour," in Swiss Transport Research Conference, 2001.
[13] S. Schönfelder and U. Samaga, "Where do you want to go today?-More observations on daily mobility," in 3rd Swiss Transport Research Conference, Ascona, 2003.

[14] J. Kurhinen and J. Janatuinen, "Geographical Routing for Delay Tolerant Encounter Networks," in Proc. of IEEE ISCC 2007, 2007, pp. 463-467.

[15] K. Miyashita, T. Terada, and S. Nishio, "A Map Matching Algorithm for Car Navigation Systems that Predict User Destination," in Proc. of AINAW 2008, 2008, pp. 1551-1556.

[16] I. Whitten and E. Frank, "Data Mining: Practical machine learning tools and techniques," 2005.

[17] A. Vahdat and D. Becker, "Epidemic routing for partially connected ad hoc networks," Citeseer, Tech. Rep., 2000.

[18] C. Maihöfer, T. Leinmüller, and E. Schoch, "Abiding geocast: time-stable geocast for ad hoc networks," in Proc. of VANET 2005. ACM New York, NY, USA, 2005, pp. 20-29.

[19] B. Karp and H. Kung, "GPSR: greedy perimeter stateless routing for wireless networks," in Proc. of MobiCom 2000. ACM New York, NY, USA, 2000, pp. 243-254.

[20] K. Kelley, "Casual Carpooling-Enhanced," Journal of Public Transportation, vol. 10, no. 4, p. 119, 2007.

[21] J. Härri, F. Filali, C. Bonnet, and M. Fiore, "VanetMobiSim: generating realistic mobility patterns for VANETs," in Proc. of VANET'06. ACM New York, NY, USA, 2006, pp. 96-97. 